\title{
Scientific Corner
}

\section{SPAT(E)US, Characterization of Small Pancreatic Tumors by (E)US $\nabla$}

Ductal adenocarcinoma of the pancreas (PDAC) is one of the leading causes of cancer related death in the western world and Japan. PDAC is the most common malignancy of the pancreas accounting for about $90 \%$ of malignant pancreatic neoplasia. In most cases (80-95\%) ductal adenocarcinoma are diagnosed at an advanced stage, with locally advanced or metastatic disease requiring palliative treatment. The 5 year survival rate of PDAC (outcome parameter) is less than 5\% which has not significantly changed over the last 30 years.

In large cohorts of pancreatic solid lesions, lesions other than ductal adenocarcinoma have been rarely reported in the past. The prevalence of metastases of other primary cancers into the pancreas and the prevalence of neuroendocrine tumors is reported to be approximately $3 \%$, respectively.

Important differential diagnoses of PDAC include

1. Neuroendocrine tumours (benign, malignant, genetically determined),

2. Metastases of renal cell carcinoma (lung, mamma, others),

3. Microcystic (and therefore solid) serous pancreatic adenoma,

4. Mesenchymal neoplasia,

5. Focal pancreatitis,

6. Others.

According to current guidelines and oncological rules all solid pancreatic lesions without contraindications to surgery (metastatic disease, age, comorbidity, locally nonresectable, amongst others) are presumed to be ductal adenocarcinoma if not otherwise proven and should be radically operated without histological or cy- tological examination prior to surgery. In addition, most guidelines so far have not recommended excluding other pathology before surgery since they are rare under normal circumstances. Not knowing the etiology before radical surgery might result in an unacceptable large proportion of patients exposed to unjustified surgery related mortality and morbidity. Confirmation of the diagnosis can be difficult and usually requires invasive procedures.

Highly sophisticated ultrasound techniques are the methods of choice to detect very small SPL.

The work up on how to differentiate between such lesions include showing that ductal adenocarcinoma are mostly hypovascular (hyperenhancing using contrast enhanced ultrasound [CEUS]) and stiffer than the surrounding pancreatic parenchyma, whereas neuroendocrine tumours (NET, benign, malignant), serous microcystic adenoma, and metastases of RCC are often hypervascular (and, therefore, hyperenhancing) and isoelastic or softer than the surrounding pancreatic parenchyma (if small).

The planned EFSUMB multicentric study focuses on the use of current available ultrasound technology in the characterisation of the histologically proven very small pancreatic lesions $\leq 15 \mathrm{~mm}$ in order to optimize the clinical management of such lesions.

We invite you to submit your cases into the EFSUMB Scientific Corner (or excel sheet directly sent to you via Christoph. Dietrich@ckbm.de). Please also prepare and submit images and videos. Would you be interested to submit your histological (cytological) proven lesions $\leq 15 \mathrm{~mm}$ (except for MEN) to the Scientific Corner [www.efsumb.org]?

\section{Pediatric Registry $\nabla$}

The Scientific Corner already contains the Pediatric Registry. In order to solve at least some of the problems related to off-label use EFSUMB has decided to create a database (EFSUMB Scientific Corner) to collect European experience in the use of CEUS in children. The database could support future spread of CEUS into pediatric daily routine use. The aim is to demonstrate safety, efficacy and efficiency. Accordingly, we would kindly ask you to submit your cases for the EFSUMB Pediatric Registry Data Base. This questionnaire aims to collect data on the use and safety of the ultrasound (US) contrast agent SonoVue ${ }^{\circledR}$ (Bracco, Italy) in children to support the joint effort of EFSUMB in the approval for its intravenous use in pediatric patients. Please, submit patient data directly via www.efsumb.org or to christoph.dietrich@ckbm.de [to be contacted for required data]. Large data can be sent via https://www.zeta-uploader.com/de (or other platforms). We also kindly ask you to encourage your colleagues in the field of pediatrics including subspecialties (e.g., pediatric surgery, pediatric nephrology and urology) who may perform CEUS in children to submit. Please support the idea that also paediatric patients will have open access to CEUS further reducing exposure to radiation. The authors are happy to be contacted for further details. 